\title{
Demanda hospitalaria pediátrica en tiempos de COVID-19
}

\section{Pediatric hospital demand in the COVID-19 pandemic}

Correspondencia

Emiliana Rizo Patron

emirizop@hotmail.com

Recibido: $12 / 06 / 2020$

Arbitrado por pares

Aprobado: 20/08/2020

Citar como: Rizo-Patron E, Padilla J, Tantaleán JA. Demanda hospitalaria pediátrica en tiempos de COVID-19. Acta Med Peru. 2020;37(3):37681. doi: https://doi.org/10.35663/ amp.2020.373.1000

\author{
Emiliana Rizo-Patron ${ }^{1, a}$, Justo Padilla ${ }^{1,2, b}$, José A. Tantaleán ${ }^{13, c, d}$ \\ Instituto Nacional de Salud del Niño San Borja. Lima, Perú. \\ Sociedad Latinoamericana de Investigación Pediátrica. Buenos Aires, Argentina. \\ Universidad Nacional Federico Villareal. Lima, Perú. \\ Médico-cirujano, ${ }^{\mathrm{b}}$ médico especialista en pediatría, ${ }^{\mathrm{c}}$ médico intensivista en pediatría, ${ }^{\mathrm{d}}$ magister en medicina
}

\section{RESUMEN}

La pandemia del COVID-19 en el Perú está causando una presión inusual a nuestros recursos hospitalarios y de cuidados críticos. A medida que la infección progresa en la población, esperamos ver un alza en los casos severos y en la demanda de unidad de cuidados intensivos (UCI) donde la capacidad de camas puede ser excedida. A pesar de que usualmente el COVID-19 causa una enfermedad poco grave en los niños, debemos estar preparados para que el numero de casos pediátricos ocasionen una sobrecarga en la capacidad de recursos hospitalarios. En un escenario de un $25 \%$ de proporción de infección acumulada en la población, podremos ver aproximadamente 891 niños críticamente enfermos que requieren hospitalización en UCI. Proponemos algunas estrategias para enfrentar la escasez de recursos de cuidados intensivos que permitan asegurar la atención de niños vulnerables con condiciones agudas y condiciones complejas que siguen incidiendo en los tiempos de pandemia.

Palabras clave: Pediatría; Infecciones por coronavirus; Cuidados críticos; Pandemias (fuente: DeCS BIREME).

\footnotetext{
ABSTRACT

The COVID-19 pandemic in Perú is causing an unusual pressure in our sanitarian and critic care resources. As the pandemics have progressed in the population, an increasing of several cases and intensive care units (ICU) demand will be expected, because of that, the hospitals capacities would be exceeded in a short place. Despite the low severity of COVID-19 cases in children, we expect that the projected number of pediatric cases could overwhelm the available pediatric capacity. Under a $25 \%$ cumulative infection rate scenario, there would be approximately 891 critically ill children requiring ICU admission. We propose several strategies to handle the concerns about shortfalls in our ability to provide pediatric ventilation and critical care support during the epidemic in Perú.

Keywords: Pediatrics; Coronavirus infections; Critical care; Pandemics (source: MeSH NLM).
} 


\section{INTRODUCCIÓN}

La expansión de la infección por coronavirus 2019 (COVID-19) presenta un reto sin precedentes para nuestro sistema de salud y para la comunidad mundial. Nuestro país debe planificar diversas estrategias para hacer frente a esta pandemia mundial, siendo una de las prioritarias el preparar a nuestras instituciones para recibir el pico de la epidemia en las mejores condiciones operativas posibles. A pesar de que la mayoría de publicaciones sobre el coronavirus del síndrome respiratorio agudo severo 2 (SARS-CoV-2) en la población pediátrica señalan la infrecuente gravedad en este grupo etario, debemos igualmente estar preparados para responder satisfactoriamente frente a esta emergencia sanitaria.

En etapas tempranas de la pandemia, se observó mayor prevalencia de COVID-19 en la población adulta y la proporción de casos confirmados en niños era relativamente pequeña. Desde entonces, debido a la propagación de contagios y la mejor capacidad de pruebas en el país, el número de casos confirmados en la población pediátrica ha aumentado significativamente, con mas de 23,000 casos y 83 muertes según el Ministerio de Salud del Perú hasta el 15 de agosto. El crecimiento en los casos, acompañado de la escasa oferta de servicios hospitalarios para esta población amerita una mayor atención a este grupo etario.

Este artículo tiene como objetivo revisar el espectro clínico del SARS-CoV-2 en la población pediátrica, proponer proyecciones del número de casos pediátricos de requerirán admisión en cuidados críticos y sugerir estrategias previniendo la posibilidad de escasez de recursos en $\mathrm{UCl}$.

\section{ESPECTRO CLÍNICO DEL SARS-COV-2 EN NIÑOS}

A diferencia de otros virus respiratorios como el de la influenza y el virus sincicial respiratorio donde la población pediátrica es considerada de riesgo, en el SARS-CoV-2 el riesgo promedio de infección grave en niños es bajo ${ }^{[1-5]}$. Uno de los primeros casos pediátricos de COVID-19 en Wuhan - China, fue un niño previamente sano de tres años que fue admitido en la $\mathrm{UCl}$ en enero 2020, recibió tratamiento con inmunoglobulinas y fue dado de alta luego de 13 días de hospitalización ${ }^{[1]}$. En una serie de casos de pacientes menores de 16 años atendidos con SARS-CoV-2 en Wuhan, encontraron que más del $60 \%$ presentó anormalidad pulmonar radiológica y el 1,8\% requirió hospitalización en la unidad de cuidados intensivos (UCI) ${ }^{[2]}$. La serie de casos pediátricos de SARS-CoV-2 más extensa fue publicada el 16 de marzo a partir de datos de China. La gran mayoría de los niños tuvo síntomas leves, y algunos fueron asintomáticos ${ }^{[3]}$. Se observó que el $10,6 \%$ de menores de un año y el $7,3 \%$ de niños entre uno a cinco años de edad, presentaron cuadro clínico grave o crítico, respectivamente. El estudio reporta una proporción general (para todas las edades) de niños con enfermedad grave o crítica de 5,8\% (Tabla 1).
A pesar de que aún el comportamiento del virus se encuentra en estudio, se considera que la presencia de un sistema inmune comprometido podría incrementar el riesgo de una enfermedad más grave. Los pacientes en tratamiento de cáncer o con condiciones médicas preexistentes como cardiopatías o enfermedades crónicas forman parte de este grupo. Aún se desconoce cuál es el riesgo de enfermedad grave por COVID-19 en niños con enfermedades preexistentes. En la serie de casos de Lu et al. (2020), los niños que requirieron cuidados críticos presentaban todos comorbilidades ${ }^{[2]}$. Por otro lado, en la influenza, la presencia de comorbilidades aumenta cerca de 6 veces la tasa de hospitalización en los pacientes pediátricos, en comparación con los niños previamente sanos ${ }^{[6]}$. Se podría interpretar que existe la posibilidad de ver una situación similar en la infección con el SARS-CoV-2, lo que debe mantenernos alerta para recibir esta demanda de pacientes.

La gravedad de las enfermedades respiratorias virales en la niñez en países en vías de desarrollo puede tener un comportamiento diferente en comparación con el resto de los países. La Organización Mundial de la Salud reporta que la gran mayoría de muertes en niños menores de cinco años por infecciones respiratorias se observan en países en vías de desarrollo, donde los niños pueden llegar en estados de gravedad más avanzados por deficiencias en atención primaria y sistemas de salud insuficientes. Esto implica, que la evidencia disponible sobre la gravedad del COVID-19 en la población pediátrica principalmente reportada de países más desarrollados, podría ser excesivamente conservadora para contextos como el nuestro. Se debe estar preparado para ver un aumento en los casos de infecciones respiratorias de todo el espectro clínico en nuestra población pediátrica, incluyendo infecciones cuya etiología es el SARS-CoV-2.

Los efectos pro-inflamatorios del SARS-CoV-2 son una fuente de preocupación ya que interviene en la presencia de complicaciones y de casos severos de COVID-19, sobre todo en adultos. Recientemente se ha reportado un nuevo síndrome asociado a la infección por SARS-CoV-2 en niños y adolescentes llamado "síndrome inflamatorio multisistémico en niños" (MIS-C por sus siglas en inglés). Las manifestaciones clínicas de esta entidad son similares a la enfermedad de Kawasaki y al síndrome de shock tóxico en el que se puede ver compromiso de dos o más órganos y enfermedad severa que requiere hospitalización ${ }^{[7]}$. La información disponible sobre la frecuencia y el curso clínico del (MIS-C) aún es limitada.

\section{PROYECCIÓN DE NÚMERO DE CASOS PEDIÁTRICOS}

A pesar de que esperamos observar una proporción baja de niños gravemente enfermos con COVID-19, al considerar la magnitud de población susceptible en el Perú, podría resultar en un número considerable de niños que requieren hospitalización o cuidados críticos. Por ejemplo, el sistema de vigilancia de COVID-19 en UCls pediátricas implementado en los Estados Unidos, reporta que hasta el 22 de abril se habían admitido 151 niños con COVID-19 en 
Tabla 1. Criterios de gravedad en casos pediátricos de COVID-19

\begin{tabular}{ll}
\multicolumn{1}{c}{ Gravedad } & \multicolumn{1}{c}{ Definición } \\
\hline Infección & No signos ni síntomas clínicos \\
asintomática & Radiografía o tomografía axial computarizada normal \\
Leve & Síntomas de infección respiratoria aguda alta (fiebre, malestar general, mialgia, tos, odinofagia, secreción \\
& nasal o estornudos). \\
& Examen físico: congestión de faringe, no anormalidades a la auscultación. \\
& Algunos casos solo presentaron síntomas digestivos (nausea, vómitos, dolor abdominal o diarrea). \\
Moderada & Signos clínicos de neumonía. \\
& Fiebre y tos frecuente. \\
& Tos mayormente seca, seguida de tos productiva \\
& Algunos pueden tener estertores (sibilantes, roncos) \\
& No hipoxemia evidente (disnea o falta de aire), \\
& Algunos casos sin síntomas o signos clínicos, pero imágenes muestran lesiones pulmonares, subclínicas. \\
& Síntomas respiratorios tempranos como fiebre y tos, puede estar acompañado de síntomas gastro- \\
Severa & Prostinales \\
& Saturación de $0_{2}<92 \%$, sin otras manifestaciones de hipoxia. \\
& Progreso rápido a síndrome de distrés respiratorio agudo o falla respiratoria \\
& Puede presentar shock, encefalopatía, injuria miocárdica o falla cardiaca, disfunción de coagulación e injuria \\
& renal aguda. Disfunción orgánica que atenta la vida. \\
\hline
\end{tabular}

Fuente: Elaboración propia, adaptado de Dong et al., (2020) [3]. Epidemiological characteristics of 2143 pediatric patients with 2019 coronavirus disease in China, Pediatrics.

unidades de cuidados intensivos pediátricos con un total de 183 instituciones participantes, cifra que se considera subestimada puesto que es un registro voluntario ${ }^{[8]}$. La disponibilidad de este tipo de datos permite modelar diferentes escenarios de población infectada. En un estudio reciente, con el objetivo de aproximar la cantidad de casos pediátricos graves y críticos totales en los Estados Unidos, Pathak et al. (2020), estimaron empíricamente la proporción de casos pediátricos graves y críticos a partir de cálculos epidemiológicos reportados hasta el momento ${ }^{[9]}$. En un escenario de 50\% de población infectada, estiman unos 10865 niños que requerirán cuidados críticos en ese país.

Bajo una premisa similar, si para el Perú, consideramos una población total de 8488761 de niños menores de 15 años, según las estimaciones del Instituto Nacional de Estadística e Informática (INEI) ${ }^{[10]}$, podemos calcular que, por cada punto porcentual adicional de población infectada, el número de niños menores de 15 años infectados se incrementaría en 8400 casos aproximadamente. Si utilizamos esa población para trazar un boceto del número de casos pediátricos menores de 15 años graves y críticos que se podrían presentar durante la epidemia en el Perú y utilizando los factores estimados por Pathak, podemos calcular que en un escenario moderado-conservador con una proporción acumulada de población pediátrica infectada del $25 \%$, podríamos observar alrededor de 5051 niños gravemente enfermos y 891 críticos. La Figura 1 describe la frecuencia de niños gravemente enfermos y críticos para distintos escenarios de población contagiada.

\section{RETOS PARA NUESTRO SISTEMA DE SALUD}

El Perú se encuentra en posiciones rezagadas en relación con nuestros países vecinos y del mundo, ubicado en el puesto 100 de gasto en salud per cápita a nivel mundial y puesto 21 en Latino América. Según el Índice de Seguridad Sanitaria Global (GHSI, por sus siglas en inglés), el Perú está medianamente preparado en seguridad sanitaria. Sin embargo, respecto a indicadores específicos sobre la capacidad de salud hospitalaria, el país está calificado como poco o no preparado con un puntaje de 6,3 (rango 0 a 100) ${ }^{[11]}$. Estar preparados para sincronizar la oferta y la demanda de servicios hospitalarios es un reto difícil de alcanzar para sistemas de salud pública en todo el mundo, en especial para países de medianos y bajos ingresos, incluso en condiciones sin la demanda adicional generada por una epidemia. Para el caso de la población pediátrica vemos que usualmente las UCls en nuestros países se encuentran principalmente en instituciones de referencia ubicados en las grandes ciudades. Los recursos disponibles para atender a pacientes críticos son limitados y están muy relacionados al gasto per cápita en salud y a la capacidad nacional de camas de hospitalización en general ${ }^{[12]}$.

Frente a las proyecciones de que la capacidad de camas hospitalarias y de UCI para la población adulta podría ser excedida en algún momento durante la epidemia en el país, la opción de ceder espacios de instituciones pediátricas para pacientes COVID-19 resulta en una posibilidad que nuestras autoridades están considerando para enfrentar la situación en el futuro. 


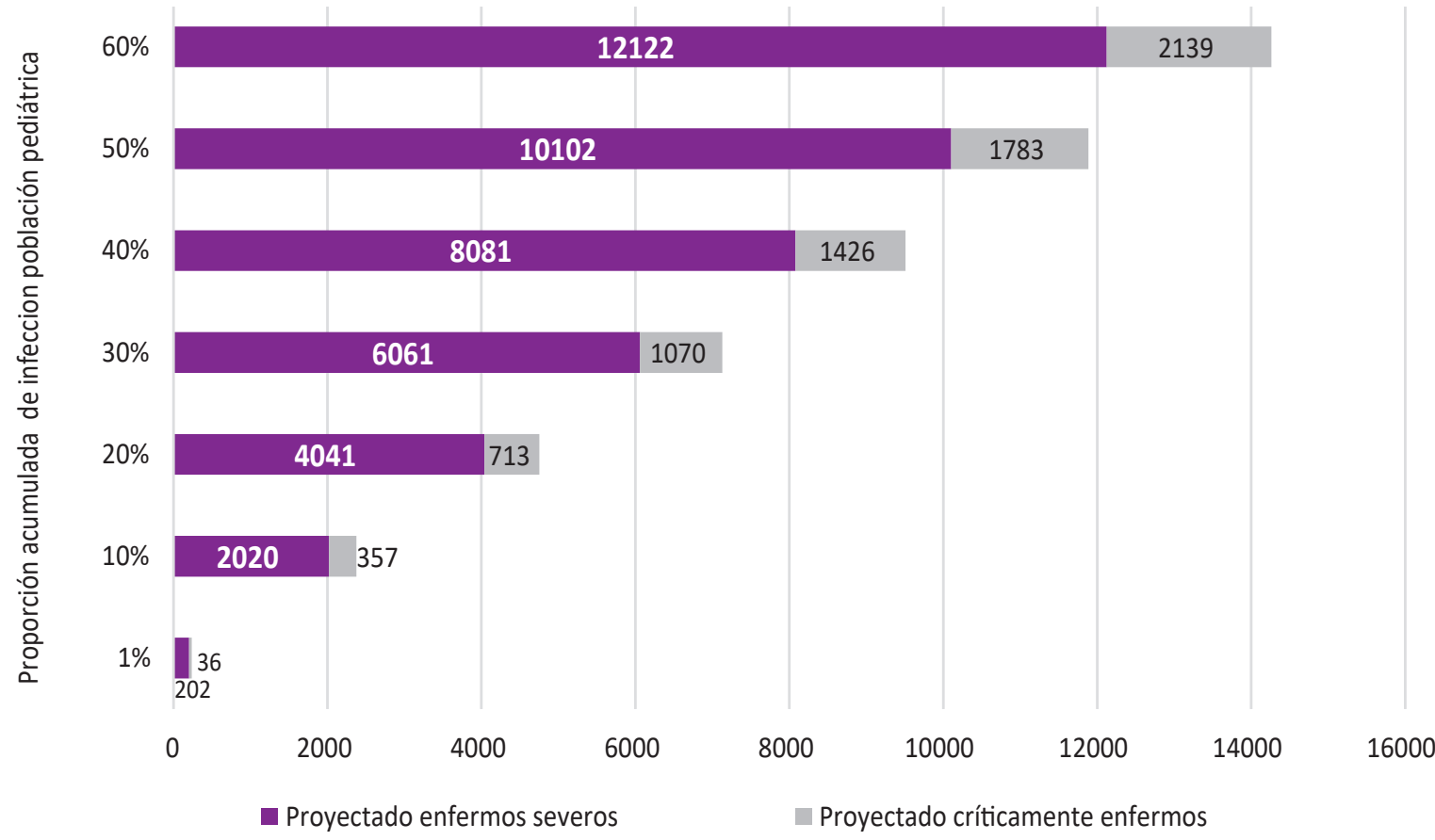

Figura 1. Número de pacientes pediátricos severos y críticos estimados COVID-19 para diferentes escenarios de infección pediátrica durante el 2020. Severos: Disnea con cianosis central. Críticos: Distrés respiratorio, insuficiencia respiratoria. Fuente: Elaboración propia, según datos del INEI ${ }^{[10]}$. Factor para enfermos severos y críticos $(0,00238 \text { y } 0,00042)^{[9]}$.

Para estimar el costo-oportunidad de ceder camas y equipos destinados para niños a la población adulta frente la emergencia, un aspecto a considerar es el tiempo promedio que cada cama estará no disponible para atender a un niño en estado crítico. Lamentablemente lo que se conoce de la enfermedad COVID-19, no permite estimar con precisión el número de días promedio de uso en cama de UCI por cada paciente. Sin embargo, según un estudio de pacientes críticos realizado en Seattle-Estados Unidos, se reporta una mediana de nueve días de hospitalización en $\mathrm{UCl}$ (rango intercuartílico 4 a 14 días) ${ }^{[13]}$. Probablemente estos tiempos estén subestimados, ya que existen diferencias en el contexto de atención, en Perú podríamos esperar días de hospitalización más prolongados al reconocer las limitaciones de nuestro sistema de salud.

Admitir adultos en hospitales pediátricos es una postura discutible ya que los equipos, protocolos, insumos, dosificación de medicamentos y el entrenamiento de personal están diseñados para la atención del paciente pediátrico, lo que podría implicar deficiencias en el servicio asistencial brindado.

\section{POSIBLES ESTRATEGIAS}

En los niños, quienes además podrían cumplir un rol importante en la transmisión del virus, las medidas de salud pública dirigidas a la prevención como el distanciamiento social, el uso universal de mascarillas y las medidas de higiene, siguen siendo la primera línea de batalla para contener y mitigar la pandemia del SARSCoV-2. Sin embargo, al ver la rápida propagación de la epidemia en nuestra población, debemos considerar todos los frentes de atención incluyendo la atención hospitalaria. En estos momentos, al estar ad portas de la máxima demanda de pacientes críticos, y previendo la posibilidad de escasez de camas y equipos en UCIP, se plantean diversas estrategias entre las cuales planteamos las siguientes:

1. Reubicar a los pacientes crónicos hospitalizados en las UCls buscando maximizar la eficiencia de nuestros recursos de UCI para atender los casos que demandan la complejidad de la atención en UCl. Los pacientes estables y estacionarios, que requieren ventilación mecánica de larga duración con fecha indefinida de extubación en un futuro cercano deben pasar a servicios de medicina o a programas de manejo domiciliario para continuar sus cuidados. Para esto se debe dotar de condiciones básicas para viabilizar la atención con un adecuado numero de personal sanitario, asegurar evaluación por un intensivista a demanda mediante interconsultas, proveer de equipos de protección personal, habilitar instalaciones eléctricas preparadas para una potencial sobrecarga eléctrica, entre otros.

2. Implementar el máximo posible de áreas en el Hospital para actuar de Terapia Intermedia. Esto ayuda a descargar a las Unidades de Cuidado Intensivo Pediátricas (UCIP) sin exponer al paciente a los riesgos de una atención básica demasiado pronto.

3. El traslado de pacientes pediátricos que ocasionalmente se encuentran en UCls de adultos a UCIs pediátricas para liberar camas en estas instituciones.

4. Ampliar la edad de admisión en UCls pediátricas a adultos jóvenes sin comorbilidades y para condiciones que son de 
manejo usual para el personal pediátrico (como trauma o sepsis).

5. La reubicación de los ventiladores mecánicos: los ventiladores mecánicos de última generación y los mas modernos disponibles deben reservarse para la $\mathrm{UCl}$, por múltiples razones. Los niños en los Servicios de Medicina, con patología pulmonar leve o inexistente que requieren soporte ventilatorio, como síndrome de Guillain Barre, traumatismos encéfalo-craneanos, encefalitis, entre otros, puede recibir soporte con equipos más sencillos que cumplen su función a cabalidad en estos casos.

6. Establecer el uso secuencial de las UCl pediátrica, de tal modo que todas puedan atender pacientes COVID-19, pero no en forma simultánea, derivando a los pacientes a "la primera UCl" y, cuando ésta se haya copado, pasar a la segunda, etc. Podría discutirse el orden de la secuencia, pero habría que considerar que los centros hospitalarios III-1 y III-2 atienden pacientes con comorbilidades y crónicos con mayor frecuencia, y este grupo de niños tiende a presentar las formas más graves de la enfermedad; asimismo, podría limitarse o al menos retrasarse el contagio.

7. Fortalecer las áreas de telesalud de las instituciones cuando no es posible el traslado de pacientes y para cubrir brechas de capacidades.

8. Consideramos que es indispensable la creación de una base de datos centralizada donde estén disponibles datos actualizados, sobre la capacidad de recursos de $\mathrm{UCl}$ y hospitalización de manera de asignar adecuada y oportuna a los pacientes críticos, utilizando esta herramienta actualizada en tiempo real optimizando la eficiencia de nuestro sistema.

9. Finalmente, a pesar de que es una medida a implementar a mediano y largo plazo, es el momento de implementar una política de capacitación para personal de atención a niños críticos. Se puede coordinar con las instituciones que las representan para identificar el número y distribución e investigar la disponibilidad para laborar en más de un lugar. En cuanto a los médicos, los intensivistas y emergencistas que no se encuentren laborando en alguna de estas áreas, podrían ser convocados en estas circunstancias. Los Residentes de tercer año y Pediatras recién egresados de la Residencia han tenido al menos 3 meses de rotación en $\mathrm{UCl}$ en los 12-24 meses previos, y si bien no pueden asumir la responsabilidad total de los pacientes, pueden servir de apoyo eficaz a los intensivistas. Las enfermeras entrenadas en atención de pacientes críticos no abundan y son una necesidad de primer orden. Conseguir el personal apropiado (en número y entrenamiento) puede ser una tarea difícil de lograr.

\section{CONCLUSIÓN}

Durante esta pandemia, hemos sido testigos de los estragos del COVID-19 en naciones con sistemas de salud sólidos. Así mismo, esta situación ha mostrado con mayor intensidad las serias deficiencias de nuestro sistema de salud. Necesitamos con urgencia un replanteamiento de la capacidad hospitalaria para el cuidado de pacientes críticos mediante una estrategia integral que incluya el gasto eficiente de recursos para obtener una oferta adecuada y suficiente de equipamiento, infraestructura y capital humano.

La responsabilidad primordial de nuestras UCls pediátricas es la atención de niños críticamente enfermos. Debemos redoblar esfuerzos para asegurar la atención de niños vulnerables con condiciones agudas y condiciones complejas que siguen incidiendo en los tiempos de pandemia, que requieren tratamiento especializado y que incrementarán la demanda en nuestras instituciones.

Agradecimiento: al Dr. Grimaldo Ramírez Cortez por su valioso aporte técnico durante la elaboración de este manuscrito.

Contribución de los autores: ERP y JP participaron en la concepción inicial de la idea y diseño del artículo. ERP, JP y JAT participaron en la redacción, la revisión crítica y la aprobación de la versión final del artículo.

Financiamiento: el presente trabajo fue financiado por el Instituto Nacional de Salud del Niño San Borja

Potenciales conflictos de interés: ninguno de los autores tiene conflicto de interés.

\section{ORCID:}

Emiliana Rizo-Patron: https://orcid.org/0000-0002-2555-3774 Justo Padilla: https://orcid.org/0000-0001-8534-4758 Jose A. Tantaleán: https://orcid.org/0000-0002-7143-4792

\section{REFERENCIAS BIBLIOGRÁFICAS}

1. Liu W, Zhang $\mathrm{Q}$, Chen J, Xiang R, Song $\mathrm{H}$, Shu S, et al. Detection of Covid-19 in Children in Early January 2020 in Wuhan, China. N Engl J Med. 2020;382(14):1370-1. doi: 10.1056/NEJMc2003717.

2. Lu X, Zhang L, Du H, Zhang J, Li YY, Qu J, et al. SARS-CoV-2 Infection in Children. N Engl J Med. 2020;382(17):1663-5. doi: 10.1056/ NEJMc2005073.

3. Eastin C, Eastin T. Epidemiological characteristics of 2143 pediatric patients with 2019 coronavirus disease in China. J Emerg Med. 2020;58(4):712-3. doi: 10.1016/j.jemermed.2020.04.006.

4. Wei M, Yuan J, Liu Y, Fu T, Yu X, Zhang Z-J. Novel Coronavirus Infection in Hospitalized Infants Under 1 Year of Age in China. JAMA. 2020;323(13):1313-4. doi: 10.1001/jama.2020.2131.

5. Zeng L, Xia S, Yuan W, Yan K, Xiao F, Shao J, et al. Neonatal EarlyOnset Infection With SARS-CoV-2 in 33 Neonates Born to Mothers With COVID-19 in Wuhan, China. JAMA Pediatr. 2020;174(7):722-5. doi: 10.1001/jamapediatrics.2020.0878.

6. Cromer D, van Hoek AJ, Jit M, Edmunds WJ, Fleming D, Miller E. The burden of influenza in England by age and clinical risk group: $A$ statistical analysis to inform vaccine policy. J Infect. 2014;68(4):36371. doi: 10.1016/j.jinf.2013.11.013.

7. Centers for Disease Control and Prevention. Multisystem Inflammatory Syndrome in Children (MIS-C) Associated with 
Coronavirus Disease 2019 (COVID-19) [Internet]. 2020 [citado 30 de julio 2020]. Disponible en: https://emergency.cdc.gov/han/2020/ han00432.asp

8. Virtual Pediatric System. COVID-19 Data: North American Pediatric ICUs [Internet]. 2020 [citado 22 de abril 2020]. Disponible en: https://covid19.myvps.org/

9. Pathak EB, Salemi JL, Sobers N, Menard J, Hambleton IR. COVID-19 in Children in the United States: Intensive Care Admissions, Estimated Total Infected, and Projected Numbers of Severe Pediatric Cases in 2020. J Public Health Manag Pract JPHMP. 2020;26(4):325-33. doi: 10.1097/PHH.0000000000001190.

10. Instituto Nacional de Estadistica e Informatica. Perú: Estimaciones y Proyecciones de Población, 1950 - 2050 [Internet]. Lima: INEl; 2001 [citado 30 de julio 2020]. Disponible en: https://www.inei.gob.pe/
media/MenuRecursivo/publicaciones_digitales/Est/Lib0466/Libro. pdf

11. Gozzer E, Canchihuamán F, Espinoza R. COVID-19 y la necesidad de actuar para mejorar las capacidades del Perú frente a las pandemias. Rev Peru Med Exp Salud Publica. 2020;37(2):371-3. doi: 10.17843/rpmesp.2020.372.5410.

12. Bhatraju PK, Ghassemieh BJ, Nichols M, Kim R, Jerome KR, Nalla AK, et al. Covid-19 in Critically III Patients in the Seattle Region Case Series. N Engl J Med. 2020;382(21):2012-22. doi: 10.1056/ NEJMoa2004500.

13. Murthy S, Leligdowicz A, Adhikari NKJ. Intensive care unit capacity in low-income countries: a systematic review. PloS One. 2015;10(1):e0116949. doi: 10.1371/journal. pone.0116949. 